\title{
影部 \\ Primeros resultados de las investigaciones arqueológicas desarrolladas en el sector central de la sierra de San Luis (Argentina)
} RMA

\author{
First results of the archaeological research conducted in \\ the central sector of the San Luis hill (Argentina)
}

VI Jornadas
Arqueológicas Cuyanas

Manuel P. Carrera Aizpitarte*

\begin{abstract}
*CONICET, Museo "Juan B. Ambrosetti", Facultad de Filosofía y Letras, Universidad de Buenos Aires; Departamento de Arqueología, Facultad de Ciencias Sociales, Universidad Nacional del Centro de la Provincia de Buenos Aires. E-mail: mcarreraaizpitarte@gmail.com
\end{abstract}

\begin{abstract}
Resumen
Se presentan los primeros resultados de las investigaciones arqueológicas iniciadas en el sector central de la Sierra de San Luis. Estos estudios se enmarcan en un proyecto mayor cuyo objetivo general es comprender las estrategias de uso del espacio implementadas por las sociedades cazadoras-recolectoras en este espacio serrano. Hasta el momento se han realizado trabajos de campo orientados a la prospección de diferentes sectores del área de estudio, el sondeo de potenciales sitios arqueológicos y el registro de estructuras fijas de molienda. La mayoría de los sitios identificados se localizan sobre la vertiente oriental de la sierra, principalmente en las inmediaciones de la localidad El Trapiche. En el caso de las estructuras fijas de molienda, se las encuentra tanto en forma aislada como en conjuntos, presentando, en general, características similares en cuanto a sus dimensiones. El registro arqueológico presentado en este trabajo constituye un primer avance en la generación de información acerca de los grupos humanos que en el pasado ocuparon este sector de la provincia.
\end{abstract}

Palabras clave: Sierras Centrales; provincia de San Luis; Cazadores-recolectores; Sitios Superficiales; Morteros Fijos.

\begin{abstract}
The first results of archaeological research conducted in the central sector of the San Luis Hill are reported in this paper. These studies are part of a larger project that has the main objective of evaluating the past hunter-gatherer land use patterns in this mountain area. So far, fieldwork has been oriented to the survey of different sectors of the study area, as well as the record of potential archaeological sites and grinding structures. Most of the identified sites are located on the eastern slope of the hill, mainly in the surroundings of El Trapiche locality. Regarding the grinding structures, they were recorded both isolated and clustered, although their dimensions are similar in both cases. Finally, the archaeological record presented here constitutes an important first step in order to generate information about the human groups that inhabited the central sector of the San Luis Hill.
\end{abstract}

Keywords: Central Hills; province of San Luis; Hunter-gatherers; Surface Sites; Grinding Structures.

La provincia de San Luis presenta dos grandes ámbitos de opuesta manifestación topográfica: el área de las serranías y el de las planicies. Siguiendo a González Díaz (1981), dentro de la primera, se distinguen: 1) el "Cordón de las Serranías occidentales", una cadena serrana baja y discontinua, ubicada al oeste de la provincia e integrada, entre otras, por las sierras de Guayaguas, de las Quijadas y del Gigante; 2) la "Sierra de San Luis", ubicada en forma de arco desde el noreste al sudoeste, constituye el macizo montañoso de mayor altura provincial; 3) al oriente, la "Sierra de los Comechingones"; 4) Diversas sierras aisladas (El Morro, La Estanzuela, Tilisarao, entre otras) localizadas en la parte central y meridional de la "Depresión Oriental". Dentro de este esquema topográfico, el área de estudio seleccionada corresponde al sector central de la Sierra de San Luis, ocupando una superficie de ca. $875 \mathrm{~km}^{2}$. Sus límites coinciden con las localidades El Molle (sudoeste), El Trapiche (sudeste), El Chañar (noroeste) y La Carolina (noreste) (Figura 1).

La Sierra de San Luis se encuentra limitada al oeste por la "Depresión longitudinal central", al este por la "Depresión oriental" y al sur por la "Planicie medanosa". Topográficamente se caracteriza por presentar una 
abrupta ladera occidental, mientras que hacia el oriente desciende en gradería escalonada hasta la depresión del Conlara (González Díaz 1981). La diferencia de altitudes (800 a 2200 msnm), sumado a la exposición y humedad disponible, dio lugar al desarrollo de una amplia diversidad de comunidades florísticas. En las quebradas, arroyos y hondonadas, la vegetación del estrato superior está compuesta por especies arbóreas y arbustivas, mientras que en el estrato inferior predominan las gramíneas y latifoliadas. Esta situación es común en alturas entre 850 y $1350 \mathrm{msnm}$. En elevaciones superiores dominan las estepas o pampas graminosas y la presencia leñosa es insignificante (Anderson et al. 1970). Siguiendo a Cabrera (1971), en este ámbito serrano estaría representado el Distrito Chaqueño Serrano. Anderson y colaboradores (1970), por otro lado, lo incluyen dentro de la unidad fitogeográfica denominada "Pastizales y bosques serranos", cuyas especies leñosas más abundantes son espinillo, garabato, tala, piquillín y chañar.

Desde el punto de vista arqueológico, la Sierra de San Luis ha sido investigada en forma discontinua, por lo que se cuenta con escasa información acerca de las antiguas poblaciones que la habitaron. En base a los objetivos propuestos en el proyecto marco, entre marzo y abril de 2015 se comenzó a prospectar aquellos lugares que potencialmente pudieran haber sido habitados en el pasado. Como resultado se identificaron sobre el extremo oriental del área de estudio, dos sitios arqueológicos en las vecindades de la localidad El Trapiche y la presencia de estructuras fijas de molienda en La Florida, Quebrada del Duraznito, y los ríos Grande y El Trapiche. La vertiente occidental de la sierra fue prospectada con menor intensidad, lo que sumado a una baja visibilidad consecuencia de la vegetación, dio como resultado un conjunto arqueológico más exiguo.

\section{Antecedentes arqueológicos en el área de estudio}

Las investigaciones arqueológicas en la provincia de San Luis se iniciaron a finales del siglo XIX y tuvieron un importante desarrollo durante las primeras décadas del siglo $X X$, decreciendo su intensidad durante la segunda mitad del mismo (González 1960). Esta situación se revierte en los últimos años, con el desarrollo de estudios sistemáticos en la región sur (Heider 2015), en el área de la sierra de La Estanzuela (Sario 2011) y en cercanías de la localidad La Toma (Curtoni et al. 2010), así como también por el re-análisis de colecciones recuperadas en décadas anteriores (Laguens et al. 2007; Sario 2008).

En lo que respecta concretamente al área de estudio, se destaca la excavación de la gruta de Intihuasi (González 1960), y de los sitios Los Pedernales, Los Morteritos, La Angostura y El Trapiche (Gambier 1998). En base a los datos obtenidos en Intihuasi, y sumado a su experiencia

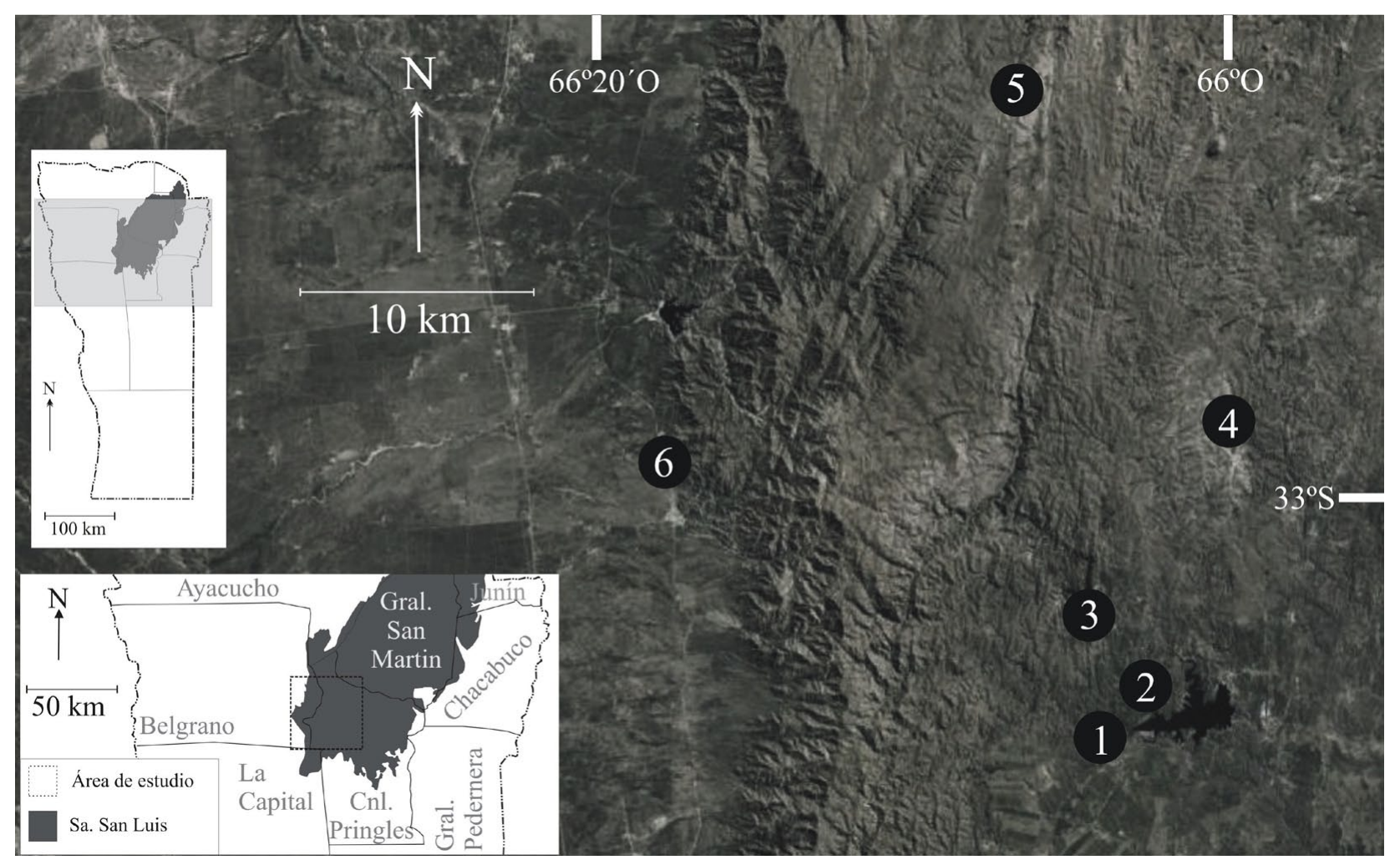

Figura 1: Área de estudio y lugares mencionados en el trabajo. Referencias: 1) Club y río El Trapiche; 2) La Florida; 3) Río Grande; 4) La Angostura; 5) Quebrada El Duraznito; 6) Villa de la Quebrada.

Figure 1: Study area and places mentioned in the paper. 1) Club and river El Trapiche; 2) La Florida; 3) Río Grande; 4) La Angostura; 5) Quebrada El Duraznito; 6) Villa de la Quebrada. 
de campo en otros sitios de Córdoba, como Ongamira y Ayampitín, González (1960) define la primera secuencia cultural para Sierras Centrales (Córdoba y San Luis). La misma postula la existencia de cuatro ocupaciones cazadoras-recolectoras: Intihuasi I, caracterizada por la presencia de puntas pequeñas y cerámica, con una edad estimada de 500 a 1500 años AP; Intihuasi II, donde ya no hay alfarería y predominan las puntas triangulares apedunculadas de tamaño mediano y grande; Intihuasi III, sin elementos absolutamente característicos, ya que presenta una mezcla de las puntas de proyectil registradas en las ocupaciones II y IV; Intihuasi IV, fechada en $7970 \pm 100$ años AP y $8068 \pm 95$ años AP, y caracterizada por la presencia de puntas lanceoladas de tipo Ayampitín.

Las excavaciones realizadas por Gambier (1998) tienen lugar entre las décadas de 1970 y 1980. En los sitios Los Pedernales, Los Morteritos y La Angostura, ubicados muy próximos entre sí, se recuperan numerosos fragmentos cerámicos y puntas de proyectil de tipo triangular. Considera que es en estos sitios donde mejor está representado el comienzo del periodo tardío de la época agropecuaria prehispánica, al que ubica cronológicamente alrededor de los 1200 años AP. Por otra parte, en El Trapiche excava dos sitios que, de acuerdo a las referencias geográficas expuestas en su publicación, se ubicaban muy cercanos a Club El Trapiche (ver más adelante). Las excavaciones dejaron de manifiesto una amplia secuencia estratigráfica de hasta $180 \mathrm{~cm}$ de profundidad, con una continuidad de fragmentos cerámicos hasta los niveles inferiores. Entre los hallazgos sobresale el esqueleto de un niño acompañado por un ajuar integrado por puntas triangulares grandes y una placa lítica con borde aserrado. Partiendo de la premisa de que la cerámica es una evidencia indirecta de la agricultura, Gambier (1998) considera que ésta última estuvo poco desarrollada en la zona de El Trapiche, debido a que la frecuencia de tiestos es significativamente inferior a la de los otros sitios mencionados en primer término.

\section{Metodología}

La mayoría del registro arqueológico analizado en este trabajo proviene de superficie. En el caso del Club El Trapiche, los materiales fueron recolectados en la base de una barranca cuyos sedimentos habían sido en parte removidos por acción antrópica reciente. Por otro lado, en las inmediaciones de Villa de la Quebrada, se recorrieron sectores del monte con menor vegetación, los cuales se manifiestan como parches aislados de escasas dimensiones. En ninguno de los dos casos señalados se pudo implementar una estrategia de prospección sistemática (i.e. transectas). Los materiales de La Angostura 1 fueron obtenidos a partir de un pequeño sondeo de 0,5 m de lado y ca. 0,3 m de profundidad, excavado por niveles artificiales de cinco centímetros.

En lo referente al análisis de las evidencias, el material lítico fue estudiado siguiendo los lineamientos propuestos por Aschero (1975, 1983) y Bellelli et al. (1985-1987). Los restos óseos fueron identificados a nivel taxonómico y anatómico, utilizando la colección de referencia de la Facultad de Ciencias Sociales (UNICEN, Olavarría). Se contabilizaron los restos y para los casos en que fue posible se calculó el Número Mínimo de Individuos (MNI, Lyman 1994). Se determinó la presencia de evidencias de procesamiento, como por ejemplo huellas de corte o fracturas antrópicas (Binford 1981; Johnson 1985). Por otra parte, se consideró una serie de variables tafonómicas tales como marcas de raíces, de roedores y meteorización, entre otras (Behrensmeyer 1978; Lyman 1994). Con respecto a las estructuras fijas de molienda, se siguió la tipología propuesta por Pastor (2015).

\section{Resultados}

\section{Club El Trapiche}

Este sitio se localiza a la entrada de la localidad homónima, en terrenos ocupados actualmente por el Club Social y Deportivo El Trapiche (3307'02.4"S/ 6603'53.2"O) (ver Figura 1). El registro arqueológico proviene de superficie y quedó expuesto como consecuencia de la remoción de sedimentos llevada a cabo en una amplia barranca de ca. $7 \mathrm{~m}$ de altura y $50 \mathrm{~m}$ de ancho, ubicada en el fondo del predio. El conjunto recuperado incluye artefactos líticos $(n=186)$, restos faunísticos $(n=25)$, humanos $(n=5)$, un artefacto óseo y dos pequeños fragmentos cerámicos. Éstos últimos presentan sus superficies alisadas, y fueron confeccionados mediante la técnica de rollo. De acuerdo a la coloración de las pastas, uno de ellos fue cocido en una atmosfera reductora, mientras que el otro denota una oxidación incompleta.

El conjunto lítico está compuesto por desechos de talla $(n=122 ; 68,5 \%)$, núcleos $(n=22 ; 12,4 \%)$, artefactos tallados y modificados por picado, abrasión y/o pulido ( $n=13 ; 7,3 \%$ y $n=21 ; 11,8 \%$, respectivamente). La materia prima más utilizada es el cuarzo $(n=140 ; 78,7 \%)$ que, excepto por los artefactos pulidos, es la de mayor frecuencia en todas las categorías artefactuales. En orden decreciente también se identificaron artefactos de rocas indeterminadas $(n=24 ; 13,5 \%)$, sílice $(n=5 ; 2,8 \%)$, granito, calcedonia y cuarcita ( $n=3 ; 1,7 \%$, cada una).

Entre los núcleos prevalecen los de tamaño grande y módulo de longitud-anchura mediano normal. La mayoría presenta planos de percusión lisos, tanto dobles como múltiples. Predominan los de tipo amorfo $(n=15 ; 68,2 \%)$, aunque también se constató la presencia de poliédricos $(n=4 ; 18,2 \%)$, prismáticos, globulosos y bipolares $(n=1$; $4,5 \%$, cada uno). En cuanto a los artefactos tallados, se identificaron preformas, bifaces, artefactos compuestos, raspadores, lascas con retoques sumarios, cepillos, cuchillos y raederas. Se destaca que ninguno de los grupos tipológicos cuenta con más de dos elementos. 
Prevalecen los fracturados sobre los enteros $(61,5 \%$ y $38,5 \%$, respectivamente), siendo éstos últimos de tamaño mediano pequeño y mediano grande, con módulo mediano normal y corto ancho, respectivamente. En relación a la confección, se registran series técnicas simples que en general se limitan al retoque marginal. Los artefactos que denotan un mayor esfuerzo de manufactura son las preformas y uno de los bifaces. Con respecto a los desechos de talla, las lascas enteras $(n=32$; $26,2 \%$ ), son en general de tamaño mediano pequeño y módulo mediano normal. En el conjunto prevalecen las de tipo angular ( $n=47 ; 58,8 \%)$ y secundarias $(n=14$; $17,5 \%)$, tendencia que se repite entre los talones, donde la mayor frecuencia corresponde a los lisos $(n=41 ; 58,6 \%)$ $y$ corticales $(n=12 ; 17,1 \%)$.

Casi la totalidad de los artefactos pulidos son manos de moler ( $n=18 ; 85,7 \%)$, de las cuales, la mayoría poseen dos caras activas $(n=12 ; 66,7 \%)$. La presencia de pigmentos minerales de tonalidades rojizas en algunas de ellas, indica que cumplieron otras funciones además de las relacionadas estrictamente con la subsistencia. Completan el conjunto un molino fracturado, un percutor y un artefacto indeterminado.

Los restos faunísticos presentan un buen estado de conservación (estadios 0 y 1 de meteorización, sensu Behrensmeyer 1978). Entre las modificaciones post-depositacionales se observan huellas de raíces y roedores. La mayoría de los restos recuperados $(n=12$; $48 \%$ ) corresponden a Lama sp., posiblemente guanaco. Otras especies identificadas son venado de las pampas (Ozotoceros bezoarticus) y vizcacha (Lagostomus maximus), todas ellas con un NMI de 1. En estos casos prevalecen los huesos largos de las patas y algunos restos craneales. Las dos especies de artiodáctilos identificadas son las únicas que presentan evidencias de procesamiento, como fracturas antrópicas, huellas de corte y alteración térmica. Por otra parte, se recuperó un instrumento óseo, de función desconocida, confeccionado sobre la diáfisis de una costilla derecha de Lama sp. La misma presenta pulido en el sector proximal y numerosas huellas longitudinales y paralelas en toda su cara lateral (Figura 2).

Entre los restos humanos se identificaron, al menos, dos individuos. Uno de ellos es un adulto que está representado por un tercer metatarso derecho, un fragmento tercio distal de fémur izquierdo, un fragmento distal de húmero derecho y un fragmento distal de tibia izquierda. El segundo individuo, del cual sólo se recuperó una diáfisis de tibia, es un infante de 2 a 4 años de edad.

\section{La Angostura}

Se localiza sobre la ruta provincial $\mathrm{N}^{\circ} 39, \mathrm{ca} .15 \mathrm{~km}$ al norte del dique La Florida. Sus coordenadas son $32^{\circ} 58^{\prime} 40.8^{\prime \prime S}$ $/ 65^{\circ} 59^{\prime} 56.8^{\prime \prime} \mathrm{O}$ (ver Figura 1). Se han identificado dos sitios arqueológicos, denominados La Angostura 1 y 2 .

\section{La Angostura 1}

Se trata de un alero de ca. $25 \mathrm{~m}$ de longitud, $7 \mathrm{~m}$ de altura y $4 \mathrm{~m}$ de profundidad desde la línea de goteo, en cuyas paredes se observan motivos abstractos y figurativos pintados en color blanco y rojo (Consens 1986). Como se mencionó en los antecedentes, este sitio fue excavado por Gambier (1998) en 1977.

El conjunto analizado está compuesto por artefactos líticos $(n=39)$, restos óseos $(n=63)$ y siete tiestos. Los mismos presentan sus superficies alisadas y la mayoría fueron cocidos en una atmosfera reductora. Sólo una de las piezas presenta decoración, la cual consiste en dos líneas semi-paralelas ejecutadas por incisión leve.

El conjunto lítico está compuesto por tres núcleos, un artefacto bifacial y 36 desechos de talla, todos ellos de cuarzo. Dos de los núcleos (piramidal y pseudo-piramidal) son de tamaño mediano pequeño y módulo mediano normal. El restante, también piramidal, es de tamaño muy grande, con módulo corto ancho. El artefacto tallado se encuentra fracturado y fue confeccionado por retoques parcialmente extendidos y marginales. Entre los desechos de talla se registra una mayor frecuencia de lascas fracturas $(n=23 ; 63,9 \%)$ que enteras $(n=6 ; 16,7 \%)$. Éstas últimas son en su mayoría de tamaño mediano pequeño y módulo corto ancho. En cuanto al tipo, prevalecen las angulares $(n=11 ; 30,6 \%)$, mientras que los talones mejor representados son los lisos $(n=5 ; 41,7 \%)$ y filiformes $(n=4$; $33,3 \%)$.

Con respecto a la identificación taxonómica de los restos óseos, casi el $40 \%$ de los elementos son indeterminados. Las únicas especies identificadas fueron Ozotoceros bezoarticus y Lama sp., de las cuales se contabilizó un número mínimo de uno y dos individuos (adulto y cría), respectivamente. El individuo adulto de Lama sp., está representado por las extremidades (fémur, falanges, autopodio), en tanto que de la cría se recuperaron falanges, una vértebra caudal y un fragmento de mandíbula. En el caso del venado, el único elemento recuperado es una epífisis distal de húmero derecho. La presencia de fracturas en estado fresco, estrías de percusión y negativos de impacto indican que los artiodáctilos probablemente fueron procesados para la extracción de médula.

\section{La Angostura 2}

Se trata de un sitio cantera-taller de cuarzo ubicado ca. $600 \mathrm{~m}$ al oeste de La Angostura 1. Alli se registran amplios afloramientos de pegmatitas que al erosionarse dejan expuesto los grandes bloques de cuarzo que la intruyen (ver Figura 2). En uno de ellos, se detectó una amplia concentración de posibles artefactos líticos, algunos de los cuales fueron recolectados para ser analizados en el laboratorio y corroborar si efectivamente eran producto 


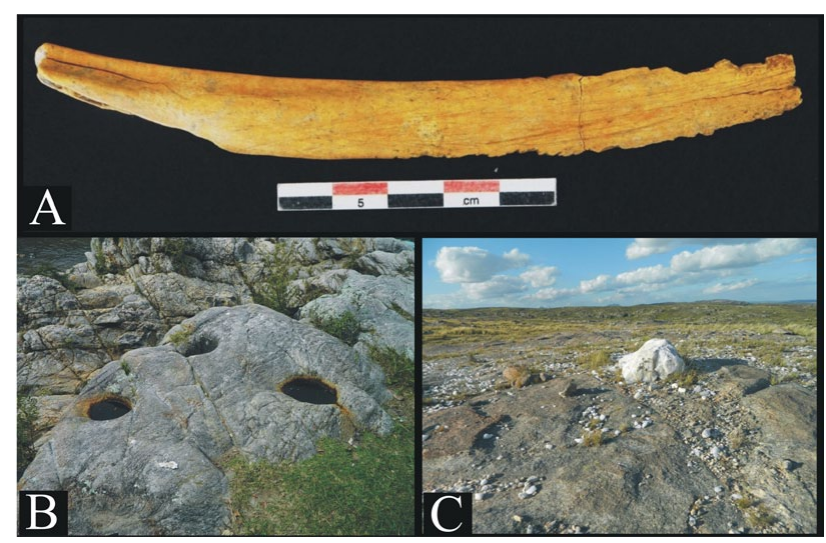

Figura 2: A) Artefacto óseo recuperado en Club El Trapiche; B) Estructuras fijas de molienda en río El Trapiche; C) Bloque de cuarzo rodeado de desechos de talla en La Angostura 2.

Figure 2: A) Bone artifact of Club El Trapiche; B) Grinding structures in river El Trapiche; C) Quartz block, surrounded of debitage in La Angostura 2.

de la acción antrópica. Dicho conjunto está formado por 52 elementos divididos en 2 núcleos, 8 instrumentos, 3 artefactos pulidos y 39 desechos de talla.

Ambos núcleos presentan características similares: son amorfos, de tamaño mediano grande, y con múltiples planos de percusión lisos. Los artefactos pulidos son los únicos confeccionados en una materia prima diferente al cuarzo. Se trata de tres manos de moler fracturadas, generadas a partir de rodados de rocas indeterminadas. La presencia de coloración rojiza sobre la superficie activa de una de ellas podría indicar funciones diferentes a las del procesamiento de recursos alimenticios. Por otro lado, entre los instrumentos tallados prevalecen las lascas con retoques sumarios $(n=3)$ y los raspadores $(n=2)$, confeccionados, en general, por medio de retoques marginales. Las piezas enteras $(n=5)$ son de tamaño grande y muy grande. En el caso de los desechos de talla se registra una mayor frecuencia de lascas enteras $(n=21$; $53,8 \%)$, entre las que predominan las de tamaño grande y módulo mediano normal $(n=9 ; 42,9 \%$ y

Tabla 1: Características de las estructuras fijas de molienda.

Table 1: characteristic of grinding structures. $\mathrm{n}=12 ; 57,1 \%$, respectivamente). Por otro lado, prevalecen las de tipo angular y los talones lisos ( $n=27 ; 84,4 \%$ y $n=13$; $48,1 \%$, respectivamente).

\section{Villa de la Quebrada}

La vertiente occidental de la Sierra de San Luis presenta una pendiente abrupta que da lugar a una planicie cubierta por monte cerrado que dificulta el tránsito y disminuye la visibilidad arqueológica. La prospección se realizó en las inmediaciones de la localidad Villa de la Quebrada (ver Figura 1) donde se recuperó un pequeño conjunto compuesto por tres desechos de talla, dos instrumentos, dos núcleos de cuarzo y un fragmento cerámico, de paredes alisadas y cocido en una atmosfera de oxidación incompleta.

Uno de los núcleos es piramidal y de tamaño muy grande, mientras que el otro fue identificado como amorfo, y es de dimensiones mediano grande. Los instrumentos consisten en dos lascas enteras (una con retoques sumarios y otra con rastros complementarios), confeccionadas en sílice y cuarzo, respectivamente. Por último, los desechos de talla (también de cuarzo) son de tipo angular, tamaño grande y módulo corto ancho.

\section{Estructuras fijas de molienda (morteros)}

Este tipo de inmuebles es abundante en Sierras Centrales, donde se los encuentra en forma aislada o formando conjuntos (ver Figura 2). En general se los considera estructuras utilizadas en la preparación de una variedad de productos, algunos de ellos vinculados con la subsistencia (Pastor 2015), aunque también se ha señalado su empleo para la obtención y acumulación de aguas meteóricas (Vignati 1931).

\begin{tabular}{|c|c|c|c|c|c|c|}
\hline Sitio & Coordenadas & $N^{\circ}$ & $\begin{array}{c}\text { Diámetro de } \\
\text { boca }(\mathrm{cm})\end{array}$ & $\begin{array}{c}\text { Forma de } \\
\text { boca }\end{array}$ & $\begin{array}{l}\text { Profundidad } \\
(\mathrm{cm})\end{array}$ & Clasificación \\
\hline \multirow{7}{*}{$\begin{array}{c}\text { La } \\
\text { Angostura } 1\end{array}$} & \multirow{7}{*}{$\begin{array}{l}32^{\circ} 58^{\prime} 40.8^{\prime \prime} \mathrm{S} / \\
65^{\circ} 59^{\prime} 56.8^{\prime \prime O}\end{array}$} & 1 & 22 & Circular & 33 & GT1 \\
\hline & & 2 & 20 & Circular & 28 & GT1 \\
\hline & & 3 & 20 & Circular & 13 & GT1 \\
\hline & & 4 & 20 & Circular & 30 & GT1 \\
\hline & & 5 & 15 & Circular & 20 & GT1 \\
\hline & & 6 & 16 & Circular & 25 & GT1 \\
\hline & & 7 & 16 & Sub-circular & 18 & GT1 \\
\hline $\begin{array}{l}\text { Quebrada El } \\
\text { Duraznito }\end{array}$ & $\begin{array}{c}32^{\circ} 50^{\prime} 35.3^{\prime \prime} \mathrm{S} / \\
66^{\circ} 6^{\prime} 08.4^{\prime \prime O}\end{array}$ & 1 & 21 & Circular & 22 & GT1 \\
\hline \multirow{5}{*}{$\begin{array}{l}\text { Alero La } \\
\text { Florida }\end{array}$} & \multirow{5}{*}{$\begin{array}{l}33^{\circ} 6^{\prime} 36.8^{\prime \prime} \mathrm{S} / \\
66^{\circ} 2^{\prime} 15.6^{\prime \prime O}\end{array}$} & 1 & 28 & Sub-circular & 25 & GT1 \\
\hline & & 2 & 30 & Circular & 38 & GT1 \\
\hline & & 3 & 26 & Sub-circular & 30 & GT1 \\
\hline & & 4 & 30 & Circular & 40 & GT1 \\
\hline & & 5 & 30 & Circular & 30 & GT1 \\
\hline \multirow{3}{*}{ Río Trapiche } & \multirow{3}{*}{$\begin{array}{l}33^{\circ} 6^{\prime} 08.3^{\prime \prime} \mathrm{S} / \\
66^{\circ} 3^{\prime} 57.9^{\prime \prime} \mathrm{O}\end{array}$} & 1 & 28 & Sub-circular & 36 & GT1 \\
\hline & & 2 & 27 & Circular & 30 & GT1 \\
\hline & & 3 & 32 & Sub-circular & 30 & GT1 \\
\hline \multirow{3}{*}{ Río Grande } & \multirow{3}{*}{$\begin{array}{l}33^{\circ} 3^{\prime} 39.6^{\prime \prime S} / \\
66^{\circ} 4^{\prime} 17.8^{\prime \prime} \mathrm{O}\end{array}$} & 1 & 20 & Circular & 40 & GT1 \\
\hline & & 2 & 28 & Sub-circular & 40 & GT1 \\
\hline & & 3 & 28 & Sub-circular & 40 & GT1 \\
\hline
\end{tabular}


En la Tabla 1 se presentan la ubicación, coordenadas geográficas, grupo tipológico (sensu Pastor 2015) y dimensiones de cada una de las estructuras documentadas. A continuación sólo se expondrán algunos aspectos específicos que no pudieron ser incluidos en la tabla mencionada. En este sentido, las estructuras $N^{\circ} 1,2$ y 3 de La Angostura 1 se ubican en el extremo norte del alero y fueron excavadas sobre su misma roca, mientras que las $N^{\circ} 4,5,6$, y 7 , se encuentran en el sector central, y fueron generadas a partir de grandes bloques desprendidos. La estructura de Quebrada El Duraznito está aislada y cercana a un pequeño alero con motivos rupestres de tipo abstracto (Consens 1986). Las estructuras $N^{\circ} 1,2,3$ y 4 de Alero La Florida se encuentran juntas y dispuestas en forma escalonada (unas encima de otras). Dentro de los conjuntos de morteros ubicados sobre las márgenes de los ríos, se destaca la disposición que presentan los de Río Grande. En este sentido, las estructuras $N^{\circ} 2$ y 3 son contiguas, mientras que la $\mathrm{N}^{\circ} 1$ se ubica en la misma roca, pero unos metros por encima.

\section{Discusión y Consideraciones Finales}

Este trabajo constituye un primer avance en el estudio sistemático de los grupos cazadores-recolectores que habitaron en el sector central de la Sierra de San Luis. Si bien la información presentada es preliminar, se exponen algunas de las tendencias generales registradas, y se discute dicha información dentro del contexto arqueológico de Sierras Centrales.

Entre los sitios analizados se observa un amplio uso del cuarzo, el cual es predominante en todas las categorías artefactuales. Su mayor frecuencia probablemente obedece a que se trata de un recurso abundante y de amplia disponibilidad, cuya adquisición no debió implicar altos costos de búsqueda. En el caso de Club El Trapiche, se lo pudo obtener en los ríos cercanos, bajo la forma de rodados, mientras que en La Angostura aflora entre las pegmatitas, bajo la forma de grandes bloques.

Entre las rocas de menor frecuencia, se identificaron cuarcita, sílice y calcedonia cuya procedencia podría ser más lejana, implicando mayores costos de adquisición. La baja proporción de desechos de talla en relación a los instrumentos y núcleos indicaría que las rocas ingresaron a los sitios en un estado avanzado de modificación, y que las actividades de talla realizadas sobre estas litologías fueron mínimas. Por otra parte, la presencia de núcleos agotados podría responder a una estrategia de extensión de la vida útil de los recursos, con artefactos que son descartados cuando ya no es posible continuar utilizándolos. En cuanto a la procedencia, Sario (2011) identifica diferentes afloramientos de sílice en la sierra La Estanzuela, distante ca. $90 \mathrm{~km}$ al este del área de estudio, mientras que Kilmurray y Villar (1981) mencionan la presencia de cuarcita feldespática en la Sierra de San Luis, aunque sin informar acerca de sus propiedades físicas.
Con respecto a la fauna, de acuerdo a Medina y Rivero (2007), durante el Holoceno temprano la dieta de las poblaciones humanas estaría basada principalmente en el consumo de camélidos, situación que se modifica a partir del Holoceno medio debido a la reducción demográfica de dicho recurso. Este nuevo escenario habría llevado a una diversificación de la dieta, incorporando especies antes no consideradas, así como también individuos inmaduros. En el caso de los sitios analizados, no se registra una amplitud en la dieta, ya que las únicas especies con evidencias de procesamiento son camélidos y cérvidos. Sin embargo, la presencia de un individuo cría de Lama sp. en La Angostura 1 podría estar respondiendo a la situación planteada por dichos autores para el Holoceno tardío. Los camélidos no solo proveyeron alimento, sino también materia prima (a través de sus huesos) para la confección de herramientas, como lo demuestra el artefacto de función desconocida recuperado en Club El Trapiche.

En los sitios considerados, la frecuencia de cerámica es mucho menor que la indicada por Gambier (1998), tanto para la zona de La Angostura como de El Trapiche. En el primero de los casos, esto puede deberse a una cuestión metodológica, de modo tal que al ampliar la excavación es posible que el número de tiestos se incremente notablemente. En El Trapiche, por el contrario, las diferencias podrían estar asociadas a otras cuestiones, como la funcionalidad de los sitios o su temporalidad. De acuerdo a los datos presentados, se considera que Club El Trapiche está vinculado a una ocupación de cazadoresrecolectores de momentos tardíos, probablemente similares a los registrados por González (1960) en el componente cultural "Intihuasi I" o por Berberián et al. (2008) en la provincia de Córdoba entre los 2000-1200 años AP.

En relación a las estructuras fijas de molienda, se observa en todos los casos presentados, una serie de características similares, vinculadas con su morfología, tipología y dimensiones. De esta manera, todas corresponden a la categoría GT11 ${ }^{1}$, presentan forma circular a semicircular, con un diámetro de boca de 25 a $30 \mathrm{~cm}$ y una profundidad que oscila entre 26 y $40 \mathrm{~cm}$. Debido a que en general se las encuentra disociadas de otras manifestaciones antrópicas, por el momento no se las puede integrar dentro de un contexto cultural y temporal determinado. En este sentido, se prevé realizar estudios específicos que permitan arrojar luz acerca de su funcionalidad.

En cuanto a las diferencias en frecuencia de hallazgos registradas entre la vertiente oriental y occidental de la sierra, se considera que la misma responde exclusivamente a problemas de muestro y no a un uso diferencial del

\footnotetext{
1 La categoría GT-1 incluye “... a los morteros profundos, útiles de boca subcircular, sección parcialmente cónica y una profundidad generalmente superior a los 10 cm" (Pastor 2015:308).
} 
espacio. En este sentido, se espera que a medida que se incrementen los trabajos de campo sobre el sector occidental, aumente el número de sitios identificados, así como también el volumen de evidencias arqueológicas.

Olavarría, 20 de febrero de 2016

\section{Agradecimientos}

Deseo agradecer a María Clara Álvarez, Ana Alcaráz, Erika Borges Vaz, Florencia Santos Valero y Gustavo Flensborg por su colaboración en el análisis de los materiales. A Paula Barros por sus útiles comentarios a una versión previa del manuscrito. Al intendente de El Trapiche, Sr. Marcelo Páez, así como también a Marcela Moiso, Juan José Nesprías, y Felix Miguel por su ayuda durante las tareas de campo. A Fátima Knott y la familia Daalgard por su hospitalidad. El único responsable de lo escrito es el autor.

\section{Bibliografía}

Anderson, D., J. Del Águila y A. Bernardon. 1970. Las formaciones vegetales en la provincia de San Luis. Revista de investigaciones agropecuarias, INTA 7(3): 153-183.

Aschero, C. 1975. Ensayo para una clasificación morfológica de artefactos líticos aplicada a estudios tecnológicos comparativos. Informe al CONICET. Ms. Buenos Aires.

Aschero, C. 1983. Ensayo para una clasificación morfológica de artefactos líticos aplicada a estudios tecnológicos comparativos. Apéndice A-C. Revisión 1983. Cátedra de Ergología y Tecnología. Facultad de Filosofía y Letras. Universidad de Buenos Aires. Ms. Buenos Aires.

Behrensmeyer, A. 1978. Taphonomic and ecologic information from bone weathering. Paleobiology 4 : 150-162.

Bellelli, C., A. Guráieb y J. García. 1985-1987. Propuesta para el análisis y procesamiento por computadora de desechos de talla lítica (DELCO- desechos líticos computarizados). Arqueología Contemporánea 2: 36-53.

Berberián, E., S. Pastor, D. Rivero, M. Medina, A. Recalde, L. López y F. Roldán. 2008. Últimos avances de la investigación arqueológica en las sierras de Córdoba. Comechingonia 11: 135-164.

Binford, L. 1981. Bones: Ancient men and modern miths. Academic Press, New York.

Cabrera, A. 1971. Fitogeografía de la República Argentina. Boletín de la Sociedad Argentina de Botánica 14(1-2): $1-42$.

Consens, M. 1986. San Luis. El arte rupestre de sus sierras. Tomo 1 y 2. Fondo Editorial Sanluiseño. San Luis.

Curtoni, R., J. Pérez \& P. Dupuy. 2010. Investigaciones arqueológicas en la provincia de San Luis. Libro de Resúmenes del XVII congreso Nacional de Arqueología Argentina. Mendoza.

Gambier, M. 1998. Arqueología de la sierra de San Luis. Instituto de Investigaciones Arqueológicas y Museo. Faculta de Filosofía, Humanidades y Arte, Universidad Nacional de San Juan, San Juan.

González, A. 1960. La estratigrafía de la gruta de Intihuasi (Prov. de San Luis, R.A.) y sus relaciones con otros sitios precerámicos de Sudamérica. Revista del Instituto de Antropología 1: 5-302.

González Díaz, E. 1981. Geomorfología. En: Geología y recursos naturales de la provincia de San Luis. Relatorio del VIII Congreso Geológico Argentino, M. Yrigoyen (ed.), pp. 193-236. Buenos Aires.

Heider, G. 2015. "Los pueblos originarios en el norte de Pampa Seca. Una mirada arqueológica a los cazadoresrecolectores del sur de las provincias de Córdoba y San Luis". Facultad de Filosofía y Humanidades. Universidad Nacional de Córdoba, Argentina. 350 páginas. Tesis Doctoral.

Johnson, E. 1985. Current development in bone technology. Advances in Archaeological Method and Theory 8: 157-235.

Kilmurray, J. \& L. Villar. 1981. El basamento de la sierra de San Luis y su petrología. En: M. Yrigoyen (ed.), Geología y recursos naturales de la provincia de San Luis. Relatorio del VIII Congreso Geológico Argentino, pp. 33-54. Buenos Aires.

Laguens, A., M. Giesso, M. Bonnin \& M. Glascock. 2007. Más allá del horizonte: cazadores-recolectores e intercambio a larga distancia en Intihuasi (provincia de San Luis, Argentina). Intersecciones en Antropología 8: 7-26.

Lyman, R. 1994. Vertebrate Taphonomy. Cambridge Manuals in Archaeology, Cambridge University Press, Cambridge.

Medina, M. \& D. Rivero 2007. Zooarqueología. Lama guanicoe y dinámica evolutiva del Chaco Serrano. Mundo de Antes 5: 211-234.

Pastor, S. 2015. Acerca de la constitución de agentes sociales, objetos y paisajes. Una mirada desde las infraestructuras de molienda (Sierras de Córdoba, Argentina). En: J. Salazar (comp.), Condiciones de posibilidad de la reproducción social en sociedades prehispánicas y coloniales tempranas en las Sierras 
Pampeanas (República Argentina), pp. 302-341. Centro de Estudios Históricos Prof. Carlos S.A. Segreti, Córdoba.

Sario, G. 2008. Tecnología bifacial en las sierras de San Luis y depresión del Conlara (provincia de San Luis, República Argentina). Arqueoweb 10: 1-18.

Sario, G. 2011. "Poblamiento humano en la provincia de San Luis: una perspectiva arqueológica a través del caso de la organización de la tecnología en Estancia La Suiza". Facultad de Filosofía y Humanidades. Universidad Nacional de Córdoba, Argentina. 289 pp. Tesis Doctoral.

Vignati, M. 1931. ¿Morteros o represas? Nueva interpretación de las agrupaciones de morteros. Notas preliminares del Museo de La Plata 1:45-61. 